\title{
Violences À Abobo (Côte d'Ivoire) Sous L'angle Du Contexte Communautaire
}

\author{
Nanan Doh N'guessan Gérard \\ Doctorant à l'UFR Criminologie \\ Université Félix Houphouët-Boigny Cocody, Côte d'Ivoire
}

Doi:10.19044/esj.2018.v14n23p100 URL:http://dx.doi.org/10.19044/esj.2018.v14n23p100

\begin{abstract}
This study focuses on analyzing inter-community violence that occurred in the urban district of Abobo (Ivory Coast) in connection with social cohesion factors. On the basis of the actionist theory, the working hypothesis states that the inter-community violence in the urban district of Abobo, is the consequence of a negative ethnocentric identity developed by some citizens against other groups of people. This behavior has been stimulated by the context of the crisis the country has gone through and by a set of sociocultural factors. One hundred and fifty (150) persons were sampled to participate in this study. The tools used for the investigation consists of documentary research, questionnaire, and observation. The qualitative and quantitative methods were used for the data processing. The results of the study show that the appearance of inter-community violence, in the urban district of Abobo, arose out of the social, political and military crisis, as well as from an atmosphere of discrimination, stigmatization, and impunity. Actually, good relations were maintained among the different communities of the district of Abobo. This relation was formerly based on mutual tolerance, forgiveness, acceptance of mutual differences and, most especially, respect for human dignity. But these relations have totally worsened and have unfortunately led to inter-community violence. Also, this violence has negatively impacted the social values that united them. This situation has broken the existing social cohesion between the people living in that urban district.
\end{abstract}

Keywords: intercommunal violence, socio-political and military crisis, sociocultural constraints, social capital, social cohesion

\section{Résumé}

L'étude a pour objectif d'analyser les violences intercommunautaires dans la commune d'Abobo (Côte d'Ivoire) en les articulant à des problématiques de la cohésion sociale. Sur la base de la théorie actionniste, 
1'hypothèse de travail est que les violences intercommunautaires dans la commune d'Abobo sont liées au développement d'une identité ethnocentrique négative, chez certains individus à l'encontre d'autres groupes d'individus dont le contexte de crise et un ensemble de facteurs socioculturels ont favorisé les agissements. Cent-cinquante (150) personnes déterminées à partir d'un échantillonnage par jugement ont participé à l'étude. Les outils d'investigation sont la recherche documentaire, le questionnaire et l'observation. Quant à l'analyse des données, le mode de traitement est qualitatif et quantitatif. Les résultats de l'étude montrent que l'émergence des violences intercommunautaires à Abobo s'explique par la crise sociopolitique et militaire, la discrimination et la stigmatisation, et l'impunité. En effet, les relations entre les communautés d'Abobo jadis basées sur la tolérance mutuelle, le pardon, l'acceptation de la différence de l'autre et surtout le respect de la dignité humaine se sont dégradées pour donner lieu à des violences intercommunautaires qui ont eu des conséquences négatives sur le capital social qui unissait les communautés. Une telle situation a conduit à la friction de la cohésion sociale des populations de cette commune.

Mots-clés: violences intercommunautaires, crise sociopolitique et militaire, pesanteurs socioculturelles, capital social, cohésion social

\section{INTRODUCTION ET PROBLÉMATIQUE}

Les sociétés sont de plus en plus confrontées à une recrudescence de la violence qui s'exprime sous des formes diverses. Qu'elle soit symbolique, psychologique ou physique, etc., elle est un phénomène universel qui détruit le tissu social et menace la vie de tous. Il n'est pas un continent, un pays, une ville, ou une communauté qui soit à l'abri (OMS, 2002). Selon l'OMS (Id.), chaque année la violence dans le monde fait plus de 1,6 million de morts.

Elle figure parmi les principales causes de décès de 15 à 44 ans, dont $14 \%$ des décès chez les hommes et $7 \%$ des décès chez les femmes. La question de la violence a été et continue d'être une préoccupation centrale pour les chercheurs en sciences humaines et sociales (Légal \& Delouvee, 2008).

\section{Les approches pluridisciplinaires de l'étiologie de la violence}

Plusieurs études sont consacrées à l'étiologie de la violence. Ces études, pour la plus part, dans une approche déterministe ont identifié plusieurs facteurs qui sont à l'origine de ce phénomène. Un premier courant de recherche s'est penché sur le phénomène de la violence d'un point de vue macroscopique, mettant en relief plusieurs approches disciplinaires. Ces approches expliquent que la violence est à l'origine des déséquilibres importants, tant au niveau individuel qu'au niveau des groupes d'individus dans nos sociétés. Les violences sont donc le signe de la friction du capital 
social des individus (Ostrom \& Ahn, 2003). Burt (1992) abondant dans le même sens qu'Ostrom et Ahn (Id) affirme que la conséquence de cette friction du capital social est la méfiance qui s'installe entre groupes d'individus, d'autant plus que les relations constitutives du capital social qui servaient à valoriser le capital humain d'un individu ou d'un groupe sont abandonnées au profit de comportements violents.

Par ailleurs, Gelles et Strauss (1988) soulignent que les facteurs intraindividuels ou certains traits de personnalité comme la faible estime de soi, le manque de contrôle de l'impulsivité ainsi que le fait de souffrir de psychopathologie sont des mécanismes réduisant les inhibitions; ce qui expliquerait les actes de violence de certains individus. Toutefois, Campbell et Landenburger (1995) soutiennent que cette approche intra-individuelle ignore le rôle du contexte social dans le choix de la violence et que par ailleurs, une majorité d'agresseurs ne souffrent pas de psychopathologie. L'origine des violences est donc à rechercher ailleurs (Campbell \& Landenburger, 1995).

Gillloz, De puy et Ducret (1997), dans une approche psychosociale, soutiennent pour leur part, que les personnes violentes ont vécu de la violence dans leur enfance. Il y aurait ainsi un apprentissage de la violence comme mode de relation dans la socialisation primaire des individus. Approche que corroborent les auteurs comme Solnit (1978), Arias (1984), et O’Leary (1988), qui stipulent que cet apprentissage se fait par l'observation des modèles aux comportements violents. Pour ces auteurs, s'il est vrai que le fait pour l'enfant d'avoir vécu ou été exposé à la violence dans sa famille d'origine le prédispose à devenir un individu violent, il est aussi avéré que tous les hommes qui ont vécu ou été exposés à de telles circonstances ne deviennent pas tous des individus violents. Kantor et Jasinsky (1998), remettant en cause la validité de l'approche psychosociale, attestent que les normes sociales et culturelles soutiendraient des inégalités de pouvoir au sein des structures familiales en favorisant un processus de socialisation apprenant aux hommes l'utilisation de la violence pour maintenir du contrôle. Huntington (1997) pour sa part, met un point d'ancrage sur la culture comme cause essentielle des violences. Il établit une corrélation entre changement, modernisation et violence. Aussi considère-il que les changements rapides dans la société favorisent la désintégration sociale et par conséquent, le développement de la violence.

\section{La violence dans le contexte urbain}

Outre ces analyses qui abordent l'étiologie de la violence dans une approche macroscopique, il existe aujourd'hui dans nos sociétés modernes une violence dite urbaine dont la résurgence actuelle est liée essentiellement à un ensemble de facteurs que nombre d'auteurs dans leur approche explicative ont tenté d'identifier. A cet effet, Pedrazzini (2005) montre que la globalisation et l'urbanisme sont les causes des violences dans nos villes. Pour 
l'auteur, la violence et l'insécurité des grandes villes sont le produit de l'incertitude née de l'accélération des changements globaux, qui selon lui diviserait les villes en fragments antagonistes entre riches et pauvres. Par contre Alain (1995), affirme que c'est la crise économique que connaît les sociétés contemporaines, qui bouleverse les mécanismes d'intégration et de socialisation d'un mode anti-individualisme et non violent.

\section{La violence dans le contexte communautaire}

Enfin, la communautarisation de la violence a de même fait l'objet d'analyses mettant en relief une série de facteurs explicatifs qu'il convient de mentionner pour comprendre la dynamique de ce type de violence. Légal et Delouvee (Op. Cit.) affirment dans leur analyse que la violence communautaire est la conséquence d'une accumulation de la frustration qui conduirait les individus à adopter des réactions violentes à l'encontre des membres d'une communauté tenue pour responsable de cette frustration. Les frustrations dont il est question sont de nature subjective et se produisent dans la mesure où les individus pensent ne pas obtenir ce à quoi ils avaient légitimement droit à cause des autres. Homer-Dixon (1994) abondant dans le même sens affirme que ce à quoi les individus croient avoir droit sont les ressources naturelles et/ou économiques rares. Cette compétition pour l'acquisition de ces ressources influence négative la qualité des relations intergroupes. Pendant cette étape d'hostilité entre groupes, les comportements agressifs conduisent à une augmentation de la cohésion intragroupe et la différentiation intergroupe. Ces différentes réactions groupales favorisent l'évolution d'un sentiment ethnocentrique. Légal et Delouvee (Op.Cit.) s'inscrivant dans cette analyse soutiennent que ce sentiment est motivé par la maximisation des intérêts des groupes en conflit. Pour Honneth (2006) la conséquence de ce sentiment ethnocentrique est la naissance de représentations défavorables induisant des comportements violents intergroupes.

Ces études bien que pertinentes, occultent la dimension actionniste des acteurs comme objet de compréhension de la violence intercommunautaire. S'inscrivant dans ce cadre, Azzi et Klein (1998) montre que la violence intercommunautaire résulte de processus individuels et sociaux qui visent à protéger et à promouvoir des identités culturelles distinctes. Ce processus individuel teinté d'ethnocentrisme va conduire selon Légal et Delouvee (Op. Cit.), à l'adoption de comportements discriminatoires intergroupes.

\section{La question et les objectifs de recherche}

Nous pouvons affirmer à la suite de ces différents courants théoriques, que toute explication de la violence est nécessairement complexe et plurifactorielle. La violence a de multiples causes. De ce point de vue, l'on est 
amené à se poser la question de savoir, pourquoi les relations entre les communautés d'Abobo jadis basées sur la tolérance mutuelle, le pardon, l'acceptation de la différence de l'autre et surtout le respect de la dignité humaine se sont-elles autant dégradées pour donner lieu à des violences intercommunautaires dans la commune d'Abobo depuis l'année 2000 jusqu'à la fin de la crise postélectorale en avril 2010?

Les objectifs poursuivis par cette étude sont de trois (3) ordres :

- Décrire les différentes formes des violences intercommunautaires ;

- Identifier les acteurs des violences intercommunautaires ;

- Analyser les déterminants de l'engagement aussi bien des communautés que des individus dans les violences intercommunautaires à Abobo.

\section{L'hypothèse et le cadre de référence théorique}

Pour atteindre ces objectifs, il sera procédé à la vérification de l'hypothèse suivante : les violences intercommunautaires dans la commune d'Abobo trouvent leur signification dans le développement d'une identité ethnocentrique négative chez certains individus à l'encontre d'autres groupes d'individus dont le contexte de crise et les pesanteurs socioculturelles ont favorisé les agissements.

Pour rendre compte de l'objet d'étude, l'on a recouru à la théorie actionniste (Cusson, 2006), qui considère la rationalité de l'individu et donc un choix stratégique de son action. Cette théorie permet d'expliquer les motivations individuelles qui soutendent les idéologies des acteurs des violences intercommunautaires dans la commune d'Abobo.

\section{MÉTHODOLOGIE}

\section{Les sites de l'étude}

L'étude porte sur les violences intercommunautaires dans la commune d'Abobo. L'enquête de terrain a été menée du 05 au 26 décembre 2017 soit sur une période de trois (03) semaines. Le choix de cette commune a été guidé d'abord par le fait qu'Abobo est une commune cosmopolite, composée d'autochtones et d'allogènes, avec une forte communauté étrangère. Mais cette diversité culturelle, qui était source d'un véritable enrichissement, est au contraire devenue source de conflits. Ensuite à cause du nombre important de sa population estimée à environ 1030658 d'habitants (RGPH, 2014) sur une superficie de 10000 ha $\left(100 \mathrm{~km}^{2}\right)$ soit une densité de 167 habitants à l'hectare (source: Mairie d'Abobo, 2014). Cette promiscuité qui jadis était synonyme de cohésion sociale s'est dégradée pour laisser place à la désunion, à la méfiance, au repli communautaire. En outre, la commune d'Abobo présentée comme une cité dortoir accueillant l'essentiel des populations à faible revenu 
de la ville d'Abidjan, ne dispose pas de tissu industriel véritable. Elle concentre une jeunesse peu qualifiée et désœuvrée, dans un contexte socioéconomique local marqué par la prépondérance de l'informel et de l'artisanat comme principales sources de revenu des ménages. Et enfin, parce que les violences intercommunautaires sont un phénomène récurrent dans ladite commune (PNUD, 2015 ; ONUCI, 2010). Pour toutes ces raisons, il apparaît opportun d'étudier ce phénomène dans cet espace pour comprendre les facteurs explicatifs de la survenue de ces violences pour que des mesures préventives idoines soient prises pour les réduire dans des proportions considérables.

En ce qui concerne le choix des quartiers de cette commune, ils ont été choisis à la fois de façon aléatoire et sur informations de personnes ressources comme étant des zones où les violences intercommunautaires se sont manifestées. Ce sont, ABOBO-BAOULE, PK 18, ANADOR, SANSMANQUE, SOGEFIA, HOUPHOUËT-BOIGNY.

\section{La population cible et l'échantillon d'étude}

Le choix de la population d'étude, a obéi à un souci de diversification des sources de données. Ce sont au total 150 personnes issues de différentes catégories sociales comme mentionnées ci-dessous qui ont été interrogées. Cette population d'enquêté a été obtenue à partir d'un échantillonnage par jugement. Le choix de cette méthode a été motivé par la liberté qu'elle offre au chercheur d'interroger les personnes présentant les caractéristiques requises pour l'enquête. Cette population concerne les personnes suivantes :

- Autorités Administratives (le maire, le sous-préfet, etc.,) : 2

- Forces de l'ordre (la police, la gendarmerie) : 10

- Chefs de communauté (leaders religieux, chef de quartiers, leaders de jeunesse etc.) :38

- Population de la commune d'Abobo : 90

- $\quad$ victimes des violences à Abobo : 10

\section{Les instruments de recueil des données}

Pour recueillir des informations sur ce phénomène, les outils d'investigation utilisés sont la recherche documentaire, le questionnaire, l'observation et 1'entretien. La recherche documentaire a fait appel aux informations recueillies dans les documents tels que les revues spécialisées, les ouvrages, les archives, les rapports (PNUD, ONUCI, CNDHCI), les articles de presse, les travaux universitaires, ainsi que les données statistiques disponibles. La recherche documentaire, bien qu'ayant été très utile dans nos recherches, a montré certaines limites que d'autres instruments de recueil des données ont corrigées, tels que l'observation, le questionnaire et l'entretien. L'observation a consisté à relever dans les réponses aux questions posées aux 
enquêtés les indicateurs de la violence (les blessures, les voies de fait et les meurtres, les assassinats, les coups volontaires, les incivilités, les injures, confrontations, bagarres, menaces, etc.) circonscrits dans l'espace et dans le temps. Aussi, le questionnaire adressé aux agents des forces de l'ordre, aux victimes des violences, etc., a été choisi pour connaitre l'expérience de vie de ces sujets vis-à-vis du phénomène. Enfin, l'entretien individuel et semidirectif a été retenu. Cette technique a l'avantage de permettre à l'enquêté de s'exprimer librement sur un sujet donné, dans les mots qu'il souhaite et dans l'ordre qui lui convient sans se disperser et sans s'écarter du sujet principal. Ainsi, lors de nos différents entretiens, nos enquêtés notamment les populations, les ONG (Commission Nationale des Droits de l'Homme (CNDHCI), etc.), les partis politiques (Rassemblement des Houphouëtistes pour la Démocratie et la Paix (RHDP), Parti Démocratique de Côte d'Ivoire (PDCI), Front Populaire Ivoirien (FPI), Rassemblement des Républicains (RDR), etc.), etc., nous ont fait part de leurs perceptions, leurs interprétations, leurs expériences, leurs inquiétudes, mais aussi, leurs besoins et proposer des solutions.

\section{Les méthodes d'analyse des données}

S'agissant du mode de traitement des données recueillies, deux types d'analyse ont été effectués : l'analyse quantitative et l'analyse qualitative. L'analyse quantitative, elle a été d'une utilité importante dans la mesure où, elle a permis de regrouper les données issues du questionnaire à l'aide de logiciel de traitement des données (usage du logiciel Statistical Package for the Social Sciences (SPSS) version 22). Par l'entremise de ce logiciel, les données collectées ont été transformées en statistiques descriptives et inférentielles. Ainsi à partir de calculs et de tableaux statistiques l'objet d'étude a été explicité quantitativement. Quant à l'analyse qualitative, elle a permis de mieux comprendre le vécu des populations en termes de significations, perceptions du phénomène étudié et de cerner les logiques des acteurs. Elle a donc permis d'analyser les données relatives aux avis, opinions, discours et idées exprimées par les personnes interviewées.

\section{LES RESULTATS DE LA RECHERCHE}

L'exposé des résultats s'articule autour de deux (2) axes, notamment les manifestations et les facteurs explicatifs des violences intercommunautaires. 


\section{* Les manifestations des violences intercommunautaires}

- La typologie des violences intercommunautaires

Il s'agit dans cette partie du travail de faire la typologie de ces violences, c'est-à-dire les décrire, et de présenter les différents acteurs de ces violences.

\section{- La distribution des violences intercommunautaires selon les personnes enquêtées}

Il ressort des résultats de l'analyse descriptive des données recueillies que sur 150 personnes interrogées, $45,5 \%$ affirment que la violence verbale constitue l'essentiel des violences intercommunautaires tandis que 22,5\% attestent que la violence physique est celle qui s'est manifestée le plus. Nous avons également $12 \%$ qui déclarent respectivement que les violences psychologique et économique constituent l'essentiel de ces violences intercommunautaires. Enfin, 8\% attestent que la violence sexuelle est celle qui s'est manifestée le plus. Ces données indiquent en général que $45,5 \%$ des enquêtés attestent que les violences intercommunautaires dans la commune d'Abobo ont donné lieu pour leur grande part à des violences verbales.

\section{- La description des violences intercommunautaires - La violence verbale}

Elle a consisté selon nos enquêtés, en des sarcasmes, insultes, hurlements, propos dégradants et humiliants, chantage, menaces, etc. C'est la violence la plus répandue. C'est ce que nous rapporte la plupart des victimes qui disent: " quand ils arrivent dans ma cour, ils commencent à prononcer des injures en paraboles, mais je ne réponds pas parce que je sais que si je réponds, ils iront chercher les gens pour me faire du mal », propos de D.A., une commerçante. La particularité de ces violences verbales, c'est qu'elles sont le fait de personnes avec lesquelles les victimes entretenaient de bonnes relations par le passé. Mais cette amitié et fraternité de longue date n'ont pas empêché de tels actes: "on n'était tous ensembles ici, quelquefois ils venaient me demander des choses, même aujourd'hui après tout ce qui s'est passé, ils continuent de venir me demander des choses et je donne le plus souvent quand je peux », propos D.F., une commerçante. Il est difficile de donner la preuve matérielle de ce type de violence car elle ne laisse pas de trace physique, elle est donc adéquate pour passer inaperçue mais ses conséquences sont aussi désastreuses à l'instar des autres types de violences (physiques, sexuelles etc.).

\section{- La violence physique}

La violence physique est la manifestation la plus visible de ces violences intercommunautaires. Elle se traduit comme tout contact physique, non désiré causant des douleurs physiques, de l'inconfort ou des blessures. 
Selon nos enquêtés, elle a constitué par exemple à donner des coups de bâtons, de pieds, de mains ou d'utiliser des armes pour frapper sa victime. Elle se traduit donc pas l'usage de la force physique pour faire mal ou pour traduire sa position de domination sur sa victime. En somme, ce sont des atteintes à l'intégrité physique occasionnant des dommages corporels visibles ou non. Ces violences ont le plus souvent engagé le pronostic vital ou laissé des séquelles permanentes sur le corps de la victime. C'est le cas de l'un de nos enquêtés qui affirme en nous montrant une cicatrice sur sa tête : " j'ai eu de la chance, ils m'ont tapé là avec une machette" propos de F.B., un agriculteur. Aussi, il nous a été rapporté le cas de certaines personnes qui ont perdu la vie : "j'ai perdu mes deux fils dans ces violences " propos de D.M, un instituteur. Comme le soulignent bon nombre des victimes que nous avons rencontrées, ce type de violence était utilisé comme moyen d'oppression, d'intimidation et surtout comme moyen d'expression des pulsions de violence que le contexte social particulier rendait récurrent et sans contrôle.

\section{- La violence psychologique}

La violence psychologique a consisté en des attitudes et propos méprisants, l'humiliation, le dénigrement, la négligence de l'autre, l'isolement imposé. Elle porte atteinte à l'estime de soi ; et permet au doute de s'installer dans l'esprit de la victime. Ce type de violence est souvent consécutif à la violence verbale et affecte psychologiquement la victime. Elle est susceptible d'occasionner un déséquilibre psychique car si les effets de cette violence ne sont pas souvent perceptibles à l'œil nu, il n'en demeure pas moins qu'ils sont lourds de conséquences et difficilement supportables et guérissables. Elle se traduit donc par une série d'attitudes méprisantes. Cette violence psychologique est le principal indicateur de risque de la violence physique. La violence psychologique s'apparente à la violence morale. Elle est relative à tout geste qui provoque la peur, réduit la dignité ou l'estime de soi. C'est pour cela qu'on parle très souvent de violence psychologique ou morale. Mais il convient de souligner que cette violence psychologique à la différence de la violence verbale qui peut avoir des effets psychologiques et constituent en des affirmations, en des dires donc en des actions positives. La violence psychologique s'est traduite par exemple par un silence accompagné de regards menaçants, de longs mouts à son aiguë ou des gestes menaçants. Toutes ces choses sont faites avec pour objectif de perturber psychologiquement la victime. A cet effet, nous avons le témoignage de l'un de nos enquêtés qui dit: "on était obligé de rester le plus souvent dans la maison. On n'avait pas la paix du cœur et je craignais beaucoup pour ma famille. Mais, je priais mon Dieu pour qu'il veille sur moi et ma famille. », propos de F.N. une commerçante. Comme nous le voyons dans cet exemple, 
les actes de violence psychologique atteignent la victime dans son être et ont parfois des conséquences sur son mode de vie.

\section{- La violence sexuelle}

Il s'agit de toute activité sexuelle non désirée ou forcée c'est-à-dire tout contact sexuel non désiré, une relation sexuelle forcée, proliférer des menaces pour obtenir une relation sexuelle. Ces violences sexuelles bien que moins répandues ont été une forme des violences intercommunautaires dans la commune d'Abobo. Elle se manifeste également dès qu'on impose à une personne, contre sa volonté, des attitudes, des paroles, des gestes à connotation sexuelle, en utilisant l'intimidation, le chantage, la violence verbale, physique ou psychologique. Ici, il s'agit généralement du viol dont le but essentiel est de jeter l'opprobre sur la partenaire dont on sait appartenir à une autre communauté avec laquelle l'on est en conflit. Ce type de violence n'a pas été très souvent évoqué par les femmes victimes, car pour elles se sont des expériences traumatisantes et indicibles. Notre seule source d'informations a été leurs proches (parents ou connaissances). Selon le témoignage que nous avons reçu d'un enquêté qui affirmait : "Quand ils sont venus d'après eux pour venger leurs frères, nous avons pris la fuite. Ma grande mère vu son âge avancé est restée avec ma servante. Ils sont venus et ont tout pris dans la maison. Ils ont aussi violé ma servante sans même prendre la peine de se protéger » propos de K.M., un agent comptable. Cette enquête nous a fait voir les conséquences qui pourraient découler de la violence sexuelle. En effet, en plus de jeter l'opprobre sur les victimes de ces violences, ces agressions sexuelles se déroulant sans protection le plus souvent, exposent ainsi ces victimes aux IST (Infections Sexuellement Transmissible), MST (Maladie Sexuelle Transmissible) et à des grossesses non désirées

\section{- La violence économique}

La violence économique s'est traduite par les actes qui ont consisté à réduire ou à éliminer l'indépendance financière de la victime et de son pouvoir de décision en matière d'argent (prendre son argent, retenir son argent, la déposséder de ces biens), etc. Selon les divers témoignages recueillis, ce type de violence a des conséquences négatives pour la victime. A cet effet, nous avons eu l'avis de l'un de nos enquêtés qui rapporte ceci : " J'avais ma boutique où je me débrouillais un peu. Mais ils venaient prendre des choses sans payer ou quelquefois ils payent la moitié, mais tu vas te plaindre où ?", propos de S.B. un commerçant. Une autre personne que nous avons interrogée à affirmer ceci : " Tous les magasins étaient fermés. Il était difficile de trouver à manger, donc ils venaient avec les armes pour voler dans les maisons des baoulé et des autres ethnies. Et là tu as la chance s'ils te volent seulement sans 
t'agresser », propos de C.G. une commerçante. Ce type de violence donnait lieu à des agressions physiques lorsque la victime opposait de la résistance.

\section{- La distribution des périodes de la manifestation des violences intercommunautaires}

Il ressort des résultats de l'analyse descriptive des données recueillies que $89,18 \%$ des enquêtés affirment que les violences intercommunautaires dans la commune d'Abobo se sont manifestées pendant la crise tandis que 4,05 $\%$ attestent qu'elles se sont manifestées avant la crise. Enfin, 6,75\% déclarent que les violences intercommunautaires se sont manifestées après la crise .Ces données indiquent en général que $89,18 \%$ des enquêtés déclarent que les violences intercommunautaires dans la commune d'Abobo se sont manifestées essentiellement pendant la crise.

\section{- La distribution des acteurs des violences intercommunautaires}

Il ressort des résultats de l'analyse descriptive des données recueillies que $46,66 \%$ de la population d'enquête affirment que les violences intercommunautaires dans la commune d'Abobo ont mis essentiellement en opposition les Malinké et les Bété tandis que 33,34\% attestent que ces violences ont opposé les Guéré et les Bété contre les ressortissants de la CEDEAO (Burkinabé, Malien, etc.). Aussi, 13,33\% des personnes interrogées déclarent que ces violences ont opposé les Malinké aux Guéré. Enfin, 6,66\% affirment que ces violences intercommunautaires ont opposé les Baoulé contre les Malinké. Ces données indiquent en général que 46,6 \% des personnes interrogées affirment que les violences intercommunautaires dans la commune d'Abobo ont mis essentiellement en opposition les Malinké et les Bété.

\section{* Les facteurs explicatifs des violences intercommunautaires - La crise sociopolitique et militaire}

Sous le règne du $1^{\text {er }}$ président de la Côte d'Ivoire, Félix HouphouëtBoigny, la Côte d'Ivoire connaissait une paix relative et était prospère à cause de l'exportation de matières premières, produites dans les zones Sud et Est, notamment le cacao, le café, etc. Cette prospérité va entrainer des migrations massives de populations des pays voisins. C'est ainsi que, notamment, les Burkinabé, les Maliens, les Guinées et bien d'autres communautés de la

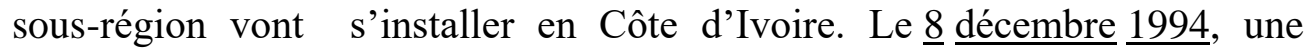
révision du Code électoral impose aux candidats à la présidence de prouver leur ascendance ivoirienne, garante de leur citoyenneté. Cette mesure va créer un malaise au sein de la population. Le 26 Août 1995, Henri Konan Bédié, alors président de la Côte d'Ivoire depuis la mort d'Houphouët-Boigny, 
introduit le concept d'ivoirité. Selon lui, ce concept permettait à la Côte d'Ivoire de mieux préserver son identité.

Mais ce concept qualifié par certains observateurs de xénophobe avait pour but d'empêcher les populations étrangères de participer à la compétition pour le pouvoir politique et d'accéder aux emplois de la fonction publique. Par ailleurs, les populations du Nord et du centre, notamment les Malinké, ont des patronymes identiques à ceux des immigrés provenant des pays voisins. C'est cette proximité culturelle qui occasionnera pour ces populations selon nos enquêtés d'être victimes de toutes sortes d'injustices. Comme le témoigne l'un de nos enquêtés, qui dit : " Certains (les nationaux ayant des patronymes identiques à ceux des immigrés provenant des pays voisins) voient leurs pièces d'identité détruites par les forces de l'ordre. Ils n'obtiennent plus de carte d'identité, de passeport et ne peuvent ni avoir de certificat de nationalité ni voter. Bien que certains Ivoiriens du Sud (notamment les Akans) qui possèdent aussi despatronymes identiques à certains peuples immigrés (Ghana, Togo et Libéria), ne subissent pas le même sort » Propos de F.B. un chef religieux. Ainsi, $u$ n sentiment de rejet va naitre chez certaines populations du Nord qui se voient assimilés à des étrangers venus des pays voisins. Cela a occasionné selon eux une classification des ivoiriens. En effet, des ivoiriens qualifiés « d'origine douteuse » sont traités de « faux ivoiriens » et appelés communément «bôrôdjan » qui signifie en langue Malinké "l'étranger ou l'homme qui vient de loin ». Ces derniers acquiescent en disant : «côbala » qui signifie en langue malinké « celui qui ne prie pas ».

En somme, cette discrimination identitaire va revêtir au fil des années plusieurs caractères notamment ethnique et religieux. Elle aura pour conséquence de créer une frustration latente qui sera exacerbée avec la crise électorale de 2010. En effet, ces élections ont contribué à diviser davantage les ivoiriens sur les questions d'obédience politique et de liberté d'expression ou de choix d'un candidat. Cette élection politique perçue par nombre d'observateurs comme étant l'élément de sortie de la longue crise qu'a connue la Côte d'Ivoire, a contre toute attente aggravé la situation de crise, à tel enseigne qu'elle a laissé apparaitre non pas une adversité politique, mais un conflit armé qui va essentiellement opposer d'un côté ceux que l'on appellera les « pro-Gbagbo » et de l'autre les «pro-Ouattara ». Cette opposition a entraîné de graves violations des droits de l'homme et troubles à l'ordre public. Ces actions seront le fait de fanatiques passionnés, que le contexte de crise aura rendu libres de toutes actions. Il convient de signaler que cette zone, sous le contrôle de la rébellion était composée en majorité des pro-Ouattara. Il était donc inadmissible pour ces derniers, dans leur zone, de permettre un quelconque soutien au président Gbagbo. Occasionnant ainsi des agressions de plusieurs communautés reconnues comme soutenant le président Gbagbo. Nous le justifions par le témoignage de M. Koffi, qui affirme : «à cause 
d'élection, les Dioula ont commencé par nous menacer pour nous interdire de voter une autre personne qu'Alassane Ouattara ». Cette situation sera marquée par l'identification de certaines ethnies comme ayant soutenues des partis politiques ou un candidat et cela aura comme conséquences d'occasionner des conflits souvent violents entre différentes communautés sur la base de préjugés. Cette situation a fragilisé la cohésion et les relations intercommunautaires.

\section{- La discrimination et la Stigmatisation}

Comme nous l'avons cité plus haut, le concept de l'ivoirité est un facteur très déterminant, dans la survenue des violences intercommunautaires en Côte d'Ivoire en général et Abobo particulièrement. Ce concept a engendré d'autres causes des violences intercommunautaires comme la stigmatisation et la discrimination ethniques. La discrimination, étymologiquement renvoie à l'action de discriminer, qui signifie effectuer un choix basé sur des formes d'inégalités qui peuvent être des caractéristiques attachées à la personne d'un individu, soit du fait d'un critère naturel (l'âge, le sexe, les origines ethniques, et), soit du fait de l'expression d'une liberté fondamentale (religieuse, politique, syndicale, etc.), soit du fait à l'accès à l'éducation ou encore à l'emploi. Elle ne permet pas de garantir à tous les individus quel que soit leur identité une égalité de traitement. Ce choix peut aussi reposer sur une double catégorisation, c'est-à-dire basée sur deux éléments tels que le groupe ethnique et la religion. La conséquence de l'introduction du concept d'ivoirité dans le jeu politique, a été de provoquer chez les populations du Nord, la croyance et la sensation de catégorisation des Ivoiriens. Cela a conduit selon eux à marginaliser, à stigmatiser et à fragiliser ceux qui s'en trouveraient, pour une raison ou pour une autre, exclus ou en être l'objet. Nous corroborons ces dires par l'affirmation de M. Soro, chauffeur à la retraite qui affirme : "on aurait vraiment pu éviter toutes ces choses, si on s'était gardé de rechercher qui était plus ivoirien que son frère. La discrimination a détruit notre fraternité, notre solidarité ». Cette discrimination et cette stigmatisation (action de blâmer publiquement) ont eu plusieurs modes d'expression soit par des actes, des paroles etc. et ont engendré des conséquences telles que le mépris, la division, la haine, le refus de collaborer.

\section{- L'impunité}

L'impunité est l'un des facteurs justifiant l'existence des violences intercommunautaires dans la commune d'Abobo. En effet, à la faveur de la crise sociopolitique et militaire qu'a connue la Côte d'Ivoire, le pays a été divisé en deux zones : la zone centre, nord et ouest (CNO) ou encore zone FN, pour signifier la partie du territoire ivoirien sous le contrôle des Forces nouvelles (FN) et la zone Sud contrôlé par le gouvernement. Mais, en ce qui 
concerne le Sud, certaines communes bien que sous contrôle gouvernemental, étaient sous l'influence de certains démembrements des Forces Nouvelles. Ce fut le cas de la commune d'Abobo, qui était sous l'emprise d'un commando dit « invisible » pendant la crise post-électorale. Ce faisant, l'administration et certaines institutions gouvernementales étaient quasi inexistantes. En l'occurrence la justice, la police, et la gendarmerie n'étaient plus en fonction. Cette situation rendait les populations plus vulnérables à l'exercice de la force, de la violence sans contrôle de la part de leurs agresseurs qui étaient assurés de l'absence d'une force de coercition en cas de commission d'actes réprimés par la loi. Selon les témoignages reçus, les éléments de ce commando encourageaient une justice tribale et partiale qui a eu comme conséquence de favoriser les agressions contre les autres communautés (baoulés, Gouro, Attié, Bété et bien d'autre, qui avaient refusé de partir ou n'avaient pas les moyens de partir). Ces agressions vont des violences verbales aux violences physiques pouvant quelquefois conduire à la mort. Comme nous le témoigne $M$. Kouadio en disant que les conditions à remplir pour être épargné de ces actes de violence ethnique étaient d': " être malinké, savoir parler le dioula, ou avoir un patronyme des groupes ethniques $d u$ Nord ». Toutes ces informations que nous avons reçues ont bien évidemment été corroborées par les chefs des communautés avec lesquels nous nous sommes entretenu.

\section{DISCUSSION}

Cette étude montre que les violences intercommunautaires dans la commune d'Abobo trouvent leur signification dans le développement d'une identité ethnocentrique négative chez certains individus à l'encontre d'autres groupes d'individus dont le contexte de crise et les pesanteurs socioculturelles ont favorisé les agissements. En effet, la crise sociopolitique et militaire, la discrimination et la stigmatisation, et l'impunité avec leurs effets sur les relations entre les différentes communautés à Abobo, ont favorisé le développement d'une identité ethnocentrique négative. Cette identité ethnocentrique est la conséquence d'une idéologique identitaire.

Ces résultats confirment les conclusions des travaux mettant un accent particulier sur l'idée de construction sociale de la communauté qui constitue le terreau de l'engagement des individus dans les arènes de la violence (Akindès, Op.Cit. ; Bayard, 1996 ; Héritier \& Wiesel, 2008). Longtemps considérée comme un havre de paix, qui garantissait la protection et la sécurité de ses membres, la Côte d'Ivoire en général, et en particulier la commune d'Abobo a été le lieu d'expérimentation des violences intercommunautaires. Plus précisément, elles ont consisté dans cette localité à voir certaines communautés terrorisées et violentées par d'autres communautés à cause de pesanteurs politiques, socioculturelles et économiques. Ces violences selon les conclusions de Collier (2000) s'expliquent par les griefs qui cachent des 
appétits comme le sentiment de reconnaissance ou en clair, le fait pour une communauté donnée de nourrir un sentiment de mépris pour une autre communauté qu'on désire réduire au silence. Honnet (2006) analysant davantage ce sentiment ethnocentrique pour comprendre le risque que représente le développement de ce sentiment pour les communautés, affirme qu'il est ressenti et partagé par certaines communautés qui se sentent victime d'une injustice ou une ségrégation économique, sociale, politique et culturelle. Il apparait selon ces éléments que les violences intercommunautaires sont pour certaines communautés le moyen de revendiquer un mieux-être ou pour une quête de profit. Cette orientation est confirmée par les travaux de Crettier (2008) qui soutient que la violence intercommunautaire répond à une quête de profit et de prestige que les communautés se disputent. Ces différentes études malgré certaines nuances confirment les résultats de cette étude.

\section{Les limites de la recherche}

Il convient cependant de prendre les résultats de cette étude avec des précautions car elle souffre de quelques faiblesses. En effet, la technique d'échantillonnage utilisée est l'échantillon par jugement (ou a priori): le choix des individus est basé sur les compétences ou la représentativité de l'individu. Etant donné que ce type d'échantillon est lié à l'appréciation du chercheur, il ne nous permet pas de généraliser les résultats. En outre le fait de n'avoir pu interroger les populations de certains quartiers d'Abobo n'ayant pas été choisis pour l'enquête peut influer sur la fiabilité de certains résultats. Cependant, la diversité des catégories sociales enquêtées attenue quelque peu ces limites.

\section{CONCLUSION}

les résultats de l'étude montre d'une part, que les violences intercommunautaires perpétrées dans la commune d'Abobo sont majoritairement d'ordre verbale $(45,5 \%)$, physique $(22,5 \%)$, psychologique $(12 \%)$, économique $(12 \%)$ et dans une moindre mesure sexuelles $(8 \%)$. D'autre part, ces résultats montrent que notre hypothèse émise se vérifie. Elle indique que la crise politique et les conséquences qui en ont résulté, ainsi que les pesanteurs socioéconomiques et culturelles ont favorisé le développement de ces violences intercommunautaires dans ladite commune. Plus exactement, elles montrent que l'instabilité politique a favorisé l'émergence de la violence entre plusieurs communautés qui jadis cohabitaient en bonne intelligence. Elles apportent aussi la preuve que ces violences ont essentiellement opposé les Malinké aux Bété, aux Baoulé, Gouro, aux Géré, etc.

Enfin, nous retenons que les violences intercommunautaires dans la commune d'Abobo ont eu de conséquences assez graves sur les populations tant au plan physique, psychologique, économique et ont entrainé des divisons, des rancœurs, un sentiment de méfiance et d'insécurité entre les 
différentes communautés. Ces diverses conséquences freinent et fragilisent davantage les efforts de réconciliation dans la commune d'Abobo.

\section{References:}

1. Akindès, F. (2004). Des origines des crises politiques récentes au sens de l'histoire en Côte d'Ivoire', Métamorphoses du politique au Nord et au Sud. Paris: Karthala. P 113-153.

2. Alain, M. (1995). L'insécurité urbaine: l'engrenage des violences, jeunes, culture de la rue et violence urbaine en Afrique, symposium d'Ibadan, IFRA-Ibadan.

3. Arias, I. (1984). «A social learning theory explication of the intergenerational transmission of physical aggression in intimate heterosexual relationships ». Thèse de doctorat non publiée, State University of New York.

4. Bayart, J. (1996). L'illusion Identitaire. Paris: Fayard.

5. Burt, R.S. (1992). Structural Holes: The Social Structure of Competition, Cambridge, Mass.,Harvard Business Press.

6. Campbell, J.C. \& Landenburger, K. (1995). «Violence against women ».Thousand Oaks (CA),

7. CNDHCI (Commission nationale des droits de l'homme). (2010). Rapport annuel 2009 : L'état des Droits de l'Homme en Côte d'Ivoire. Abidjan : CNDHCI.

8. Collier, P. (2000). Economic Causes of Civil Conflict and Their Implications for Policy. Washington D.C: Working Paper. The World Bank.

9. Crettier, X. (2008). Les formes de la Violence. Paris : La Découverte.

10. Cusson, M. \& Meyrand, R. (2006). « Comment devient-on délinquant ?» Article de la rubrique « Le point sur... » in « Comment devient-on délinquant », mensuel n¹76, novembre.

11. Gelles, R.J. \& Strauss, M.A. (1988). Intimate Violence. The Causes and Consequences of Abuse in the American Family, Touchstone Book, Simon and Shuster Inc. p: 59-60.

12. Ghiraedello, J. M., Dupuis, et al. (2002). Les effets des politiques de lutte contre les discriminations, Politiques sociales et croissance économique. Paris : L'Harmattan. p: 395-407.

13. Gillioz, L., De Puy, J., \& Ducret. (1997). Domination et violence envers la femme dans le couple. Lausanne: Payot.

14. Héritier, F. \& Wiesel, E. (1998). Le soi, l'Autre et l'Intolérance. Paris: Grasset.

15. Homer-Dixon, F. T. (1994). Environmental Scarcities and Violent Conflict: Evidence from Cases. International Security 19 (1(Summer)): 5-40. 
16. Honneth, A. (2006). La société Du Mépris : Vers une Théorie Critique. Paris : La Découverte.

17. Huntington, S. (1997). Le Choc des civilisations, Paris : Odile Jacob. $\mathrm{p}: 293$.

18. Kantor, GK. \& Jasinski, JL. (1998). Dynamics and risk factors in partner violence. A comprehensive review of 20 years of research. Thousand Oaks.

19. Légal, J-B. \& Delouvee, S. (2008). Stéréotypes, préjugés et discrimination. Paris : Dunod.

20. Mairie D'Abobo (2014). Diagnostic de l'insécurité dans la commune d'Abobo, 30pages.

21. O'Leary, K. D. (1988). "Physical aggression between spouses. A social learning theory perspective ». New York, Plenum Press. P: 3156.

22. OMS (2002). Rapport sur la violence et santé. Genève.

23. ONUCI (2010). Rapport sur les violations des droits de l'homme et droit international humanitaire en Côte d'Ivoire.

24. Ostrom, E. \& Ahn, T.K. (2003). Foundations of Social Capital. Edward Elgar.

25. Pedrazzini, Y. (2005). La violence des villes. Paris : Cérès production.

26. PNUD (2015). «Obstacles à la cohésion sociale et dynamiques de violence impliquant les jeunes dans l'espace urbain, les voix des populations des communes d'Abobo, Treichville et Yopougon dans le district d'Abidjan ».

27. Rapport de la Direction générale des Droits de l'Homme et Etat de Droit du conseil de l'Europe (2011). Sage Publications, p : 407-425.

28. RGPH (Recensement Général de la Population et de l'Habitat) 2014. 